\title{
COMMUNICATIONS
}

\section{Using Mathematics at AIM to Outwit Mosquitoes}

\section{József Z. Farkas, Stephen A. Gourley, Rongsong Liu, and Abdul-Aziz Yakubu}

Wolbachia (Figure 1) is a reproductive parasite that infects arthropod species, including mosquitoes, all over the world. Only infected females can pass on Wolbachia infection to their offspring, and therefore Wolbachia has evolved to maximise its spread by manipulating reproductive processes to enhance the production of infected females. These manipulations include feminisation (resulting in genetic males developing as females), cytoplasmic incompatibility (which prevents Wolbachia-infected males from successfully mating with females that do not have the same Wolbachia type), and male killing (which results in increased food availability for surviving female progeny). However, it is also known that Wolbachia can block or reduce replication of viruses of mosquito-borne diseases such as dengue fever and West Nile virus (WNv). What if Wolbachia infection could be used as a biological control tool to fight mosquito-borne diseases such as WNv?

The four authors met as an AIM SQuaRE (Structured Quartet Research Ensemble), in which groups of four to six mathematicians spend a week at AIM in San Jose, California, for up to three consecutive years. Using Wolbachia to control vector-borne

József Z. Farkas is reader of applied mathematics at the University of Stirling, United Kingdom. His email address is jozsef.farkas@stir.ac.uk.

Stephen A. Gourley is professor of mathematics at University of Surrey, United Kingdom. His email address is s.gour1ey@surrey.ac.uk.

Rongsong Liu is associate professor of mathematics at the University of Wyoming. Her email address is Rongsong. Liu@uwyo.edu.

Abdul-Aziz Yakubu is professor of mathematics at Howard University. His email address is ayakubu@ howard.edu.

For permission to reprint this article, please contact: reprint-permission@ams.org.

DOI: http://dx.doi.org/10.1090/noti1340

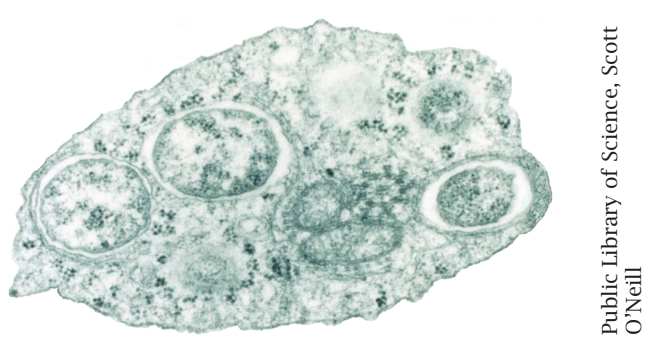

Figure 1. Transmission electron micrograph of Wolbachia within an insect cell.

diseases is a well-established idea, with field trials already under way. The main goal of our project was to theoretically investigate this possibility for West Nile virus by introducing and analysing a 12-dimensional dynamical system. As the first building block, we derived from basic principles a sex-structured model for a mosquito population infected with Wolbachia, capturing most of the key reproductive effects of the Wolbachia infection together, including male killing, in one model. The four differential equations, variables, parameters, and coefficient functions appearing in the model are given below:

$$
\begin{aligned}
M^{\prime}(t)= & -\mu_{m} M+\frac{\lambda\left(F_{\text {total }}\right)}{N}(M F+(1-\beta)(1-\tau) \\
& \left.\cdot\left(M F_{w}+M_{w} F_{w}\right)+(1-q) M_{w} F\right), \\
F^{\prime}(t)= & -\mu_{f} F+\frac{\lambda\left(F_{\text {total }}\right)}{N}(M F+(1-\beta)(1-\tau) \\
& \left.\cdot\left(M F_{w}+M_{w} F_{w}\right)+(1-q) M_{w} F\right), \\
M_{w}^{\prime}(t)= & -\mu_{m w} M_{w}+\frac{\lambda\left(F_{\text {total }}\right)}{N}(1-\beta) \tau(1-\gamma) \\
& \cdot\left(M F_{w}+M_{w} F_{w}\right), \\
F_{w}^{\prime}(t)= & -\mu_{f w} F_{w}+\frac{\lambda\left(F_{\text {total }}\right)}{N}(1-\beta) \\
& \cdot \tau\left(M F_{w}+M_{w} F_{w}\right) .
\end{aligned}
$$


- $M, F$ : numbers of uninfected male, female mosquitoes.

- $M_{w}, F_{w}$ : numbers of Wolbachia-infected male, female mosquitoes.

- $M_{\text {total }}=M+M_{w}, F_{\text {total }}=F+F_{w}, N=$ $M_{\text {total }}+F_{\text {total }}$ : total numbers of male, female, all mosquitoes.

- $\beta$ : reduction in reproductive output of Wolbachia-infected females.

- $\tau$ : maternal transmission probability for Wolbachia infection.

- $q$ : probability of cytoplasmic incompatibility (CI).

- $\gamma$ : probability of male killing (MK) induced by Wolbachia infection.

- $\lambda\left(F_{\text {total }}\right)$ : average egg-laying rate, which depends on the total number of female mosquitoes.

- $\mu_{m}, \mu_{f}$ : per capita mortality rates for uninfected male, female mosquitoes.

- $\mu_{m w}, \mu_{f w}$ : per capita mortality rates for Wolbachia-infected male, female mosquitoes.

Our rigorous analysis of the above Wolbachia model revealed, amongst other things, that under certain biologically relevant assumptions, our model has multiple steady states in which Wolbachia-infected mosquitoes could coexist with small numbers of uninfected mosquitoes.

Building on initial results in [2] and the first part of [1], we extended our mosquito population model to include WNv, which is spread by birds and mosquitoes. Our full model takes the form of a 12-dimensional system of nonlinear differential equations. We were motivated by results recently reported by Hussain et al. [3], which suggest that a particular strain of Wolbachia substantially reduces $\mathrm{WNv}$ replication in the mosquito species Aedes aegypti. We modelled this crucial phenomenon by incorporating a small parameter, the reciprocal of which is proportional to the time spent in the WNv-exposed class for Wolbachia-infected mosquitoes. This enabled us to assess the potential of Wolbachia infection to eradicate $\mathrm{WNv}$ via its effect on WNv replication in Wolbachia-infected mosquitoes. Notably, the expression we obtained for the basic reproduction number suggests that Wolbachia infection substantially reduces $\mathrm{WNv}$ replication in mosquitoes and that $\mathrm{WNv}$ will be eradicated if at the steady state the overwhelming majority of mosquitoes are infected with Wolbachia.

Wolbachia infection in mosquitoes could have a beneficial effect on the control of many other mosquito-borne diseases besides WNv. Our model of Wolbachia infection should be suitable for application to the study of whole classes of these diseases. Our ongoing work focuses on the broad application of our Wolbachia model to other mosquito-borne diseases that affect humans, such as dengue fever.

\section{References}

[1] J. Z. FARKAS, S. A. Gourley, R. LiU, and A.-A. YAKUBU, Modelling Wolbachia infection in a sexstructured mosquito population carrying West Nile virus, arXiv.org/abs/1509.06970

[2] J. Z. FARKAS and P. HINOW, Structured and unstructured continuous models for Wolbachia infections, Bulletin of Mathematical Biology 72 (2010), 20672088. arXiv.org/abs/0906.1676

[3] M. HUSSAIN ET AL., Effect of Wolbachia on replication of West Nile virus in a mosquito cell line and adult mosquitoes, Journal of Virology 87 (2013), 851-858. http://jvi.asm.org/content/87/2/851.fu11

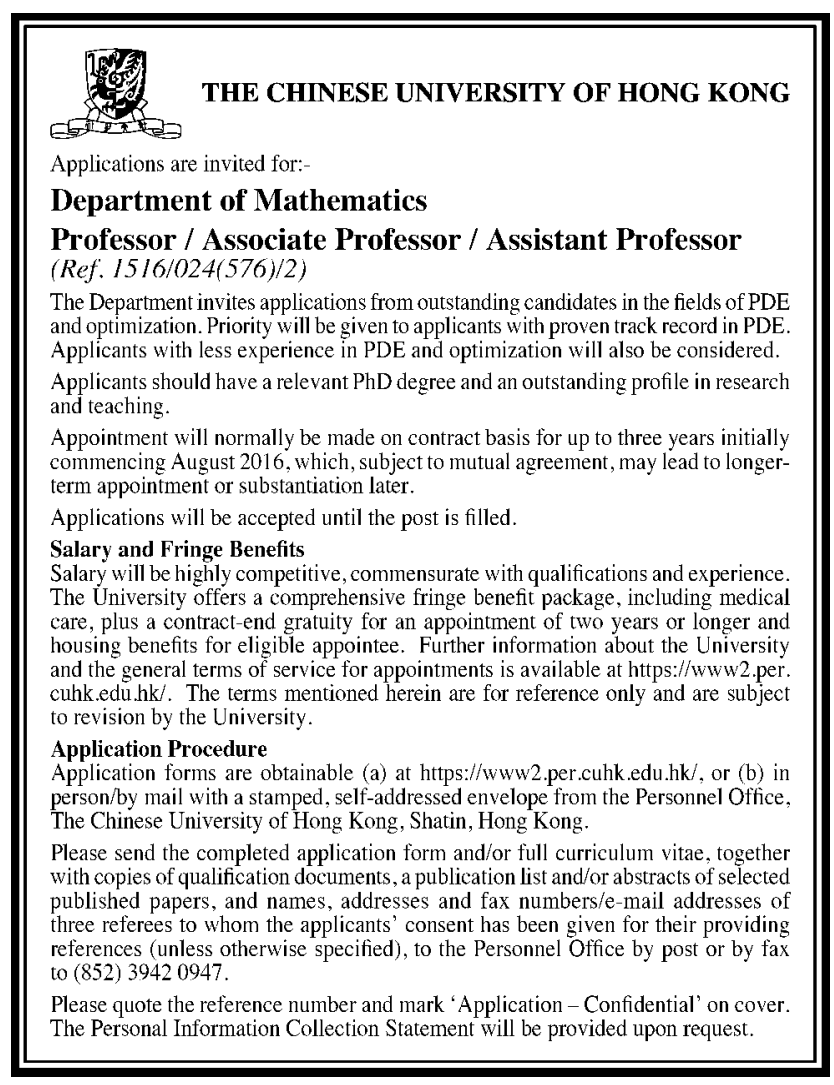

